\title{
Scanning patterns of faces do not explain impaired emotion recognition in Huntington disease: evidence for a high level mechanism
}

\section{Marieke van Asselen ${ }^{1}$, Filipa Júlio ${ }^{1,2}$, Cristina Januário ${ }^{2}$, Elzbieta Bobrowicz Campos ${ }^{3}$, Inês Almeida ${ }^{1}$, Sara Cavaco ${ }^{4}$ and Miguel Castelo-Branco ${ }^{1}$}

${ }^{1}$ Visual Neuroscience Laboratory, Faculty of Medicine, Institute of Biomedical Research in Light and Image, University of Coimbra, Coimbra, Portugal

2 Department of Neurology, University Hospital Coimbra, Coimbra, Portugal

${ }^{3}$ Faculty of Psychology and Educational Sciences, University of Coimbra, Coimbra, Portugal

${ }^{4}$ Laboratory of Neurobiology of Human Behavior, Centro Hospitalar do Porto-Hospital de S. António, Porto, Portugal

\section{Edited by:}

Marina A. Pavlova, Eberhard Karls

University of Tübingen, Germany

\section{Reviewed by:}

Golijeh Golarai, Stanford University, USA

Pia Rotshtein, University of

Birmingham, UK

\section{*Correspondence:}

Marieke van Asselen, Faculty of Medicine, Institute of Biomedical Research in Light and Image, Azinhaga de Santa Comba - Celas, 3000-354 Coimbra, Portugal. e-mail: masselen@fmed.uc.pt
In the current study, we aimed to investigate the emotion recognition impairment in Huntington's disease (HD) patients and define whether this deficit is caused by impaired scanning patterns of the face. To achieve this goal, we recorded eye movements during a two-alternative forced-choice emotion recognition task. HD patients in pre-symptomatic $(n=16)$ and symptomatic $(n=9)$ disease stages were tested and their performance was compared to a control group $(n=22)$. In our emotion recognition task, participants had to indicate whether a face reflected one of six basic emotions. In addition, and in order to define whether emotion recognition was altered when the participants were forced to look at a specific component of the face, we used a second task where only limited facial information was provided (eyes/mouth in partially masked faces). Behavioral results showed no differences in the ability to recognize emotions between pre-symptomatic gene carriers and controls. However, an emotion recognition deficit was found for all six basic emotion categories in early stage HD. Analysis of eye movement patterns showed that patient and controls used similar scanning strategies. Patterns of deficits were similar regardless of whether parts of the faces were masked or not, thereby confirming that selective attention to particular face parts is not underlying the deficits. These results suggest that the emotion recognition deficits in symptomatic HD patients cannot be explained by impaired scanning patterns of faces. Furthermore, no selective deficit for recognition of disgust was found in pre-symptomatic HD patients.

Keywords: Huntington's disease, emotion recognition, eye movements, scanning patterns of faces

\section{INTRODUCTION}

Huntington's disease (HD) is a rare neurodegenerative genetic disorder that results in motor deficits such as chorea, rigidity, and abnormal posturing. Although these motor symptoms are the most obvious manifestations of the disease, HD patients also suffer from several cognitive and behavioral abnormalities, including memory and executive impairments, as well as depression, apathy, and other psychiatric manifestations. Motor symptoms are used to define the onset of the disease, although genetic testing allows identification of the carrier state before manifestation of motor symptoms.

Sprengelmeyer et al. (1996) showed that HD patients have a reduced ability to recognize emotions in faces, in particular disgust. Additionally, Gray et al. (1997) found that pre-symptomatic HD patients also have a selective deficit for recognition of disgust. Neuroimaging studies have shown a volume reduction in the caudate of the basal ganglia in pre-symptomatic HD patients (Harris et al., 1999; Thieben et al., 2002), although some studies have indicated some subtle cortical abnormalities as well (Thieben et al., 2002; Gómez-Ansón et al., 2009). These findings led to the idea that the basal ganglia are specifically involved in recognition of disgust. However, not all studies found support for the selective deficit for recognition of disgust in HD patients (reviewed in Henley et al., 2011). For example, Milders et al. (2003) demonstrated no emotion recognition deficit in pre-symptomatic HD patients, whereas symptomatic HD patients were impaired in recognizing sadness, anger, disgust, and fear. Furthermore, Johnson et al. (2007) found that pre-symptomatic HD patients were significantly impaired on recognition of all negative emotions.

It remains unclear what is the origin of the emotion recognition deficit that is found in HD patients. Some researchers have shown that emotion recognition is not only impaired in the visual domain, but also in other domains, including auditory perception. Sprengelmeyer et al. (1996) demonstrated that HD patients were not only impaired in recognizing disgust in faces, but also from voices. This suggested a "cross-modal" deficit for disgust recognition (Calder et al., 2000). However, Sprengelmeyer et al. (2006) found that pre-symptomatic HD patients were impaired on a facial emotion recognition task, but not on the recognition 
of vocal emotions. Another interesting possibility is that HD patients have a different way of scanning faces, thereby not taking advantage of all the facial information that can be used to identify an emotion. This hypothesis was previously studied in a patient (SM) with damage to the amygdala, who has particular difficulty with recognition of fear in facial expressions (Adolphs et al., 1994). It was shown that this patient makes very few fixations to the eyes region of a face (Adolphs et al., 2005). Since the eyes region is particularly important for recognition of fear (Smith et al., 2005), this abnormal scanning pattern results in a selective deficit for fear recognition. In fact, when this patient was specifically instructed to look at the eyes region, this selective deficit disappeared. In a recent study with the same patient SM, Kennedy and Adolphs (2010) further refined this hypothesis by indicating that the amygdala is not so much involved in scanning the eyes region of a face, but is rather essential for directing gaze to most socially salient parts of the face, which are the eyes. The importance of the amygdala in visual search of facial information and therefore emotion recognition leads to the question whether the emotion recognition impairment in HD patients might also result from the way patients search and use information from a face. This is particularly interesting considering the possible role of the basal ganglia in eye movement control (Shires et al., 2010) and the finding that both pre-symptomatic and early stage HD patients have several oculomotor impairments. More specifically, it was found that HD patients demonstrated a marked delay in the initiation of volitional saccades (Blekher et al., 2004) and slower and more irregular visual scanning patterns when performing cognitive tasks such as the digit symbol subscale (Blekher et al., 2009). It was concluded that at least part of the deficit that was found on the digit symbol subscale was due to alterations in the visual scanning pattern. In case of recognition of disgust, it could be argued that the deficit that is found in HD patients is also due to impaired scanning of the faces. Since the mouth and nose region of the face are the areas with most information regarding recognition of disgust (Smith et al., 2005), it might be possible that HD patients do not look as much to this area of the face.

In the current study, we aimed to further investigate the emotion recognition impairment in HD patients and more specifically to understand whether this deficit is caused by impaired scanning patterns of the face. Therefore, we used a forced-choice emotion recognition task, including the six basic emotions. Eye movements were measured to define the number of fixations and total fixation duration to the eyes and mouth region of the face during this task. In a second task, subjects were asked to recognize emotions using only a part of the facial information, either the eyes or the mouth and nose region. This allowed us to understand if differences between patients and controls depend on controlling the face regions the participants were forced to look at. For example, if HD patients have a tendency to ignore information from the mouth region, forcing them to look at that area might improve their performance on an emotion recognition task. Finally, in order to determine if these deficits were already present at the early stages of the disease, where we can find only very specific brain lesions, we included both pre-symptomatic and symptomatic HD patients.

\section{MATERIALS AND METHODS PARTICIPANTS}

We tested 9 symptomatic patients with HD that were in an early stage of the disease, 16 pre-symptomatic HD patients, and 22 healthy control subjects. Patients were seen by an experienced neurologist and diagnosis was confirmed by genetic testing. Mean CAG repeats for the pre-symptomatic and symptomatic HD patients are reported in Table 1. The three groups were matched for education level $(F(2,44)=2.1)$. One-way ANOVA demonstrated an overall difference between the age of the two patient groups and the controls. However, Post hoc analyses indicated no age difference between the controls and pre-symptomatic HD patients and the controls and early stage HD patients. A significant difference was found, as expected, between the pre-symptomatic and symptomatic HD patients. Considering the progressive nature of the disease, this difference was unavoidable. Patient characteristics are shown in Table 1.

\section{NEUROPSYCHOLOGICAL TESTING}

To study overall cognitive functioning of the three groups, we used an extensive neuropsychological test battery. The results of these neuropsychological tests were also published in a recent paper on implicit learning in HD patients (Van Asselen et al., in press). The 12-item short form of the Raven advanced progressive matrices (Raven et al., 1993) and the vocabulary of the WAIS-III (Wechsler, 1997, 2008) were used as an indication of intelligence. Visual perception was tested with the Rey complex figure test (Rey, 1941; Osterrieth, 1944), the Benton visual retention test (Benton, 1974), and the Benton visual form discrimination test (Benton et al., 1983). The Benton facial recognition test (Benton et al. (1983) was included as a standardized test of face recognition. Verbal memory was tested using a Portuguese version of the Rey auditory verbal learning test (Rey, 1964; Cavaco et al., 2008). To assess working memory and executive functioning we used the trail making test and the digit symbol subtest of the WAIS-III and the Corsi block tapping task (Berch et al., 1998; Kessels et al., 2000). For the latter task, the product of the total number of correct trials and the length of the largest sequence was calculated (Kessels et al., 2000). The dementia rating scale-2 (Mattis et al., 2002) and the Beck depression inventory (Beck et al., 1961; portuguese adaptation: Vaz Serra and Pio Abreu, 1973a,b) were used to test for dementia and depression subsequently. Handedness was defined by using a translated version of the Edinburgh handedness inventory (Oldfield, 1971). Informed consent was obtained according to the declaration of Helsinki and all procedures were approved by our local ethics committee.

\section{FACIAL EXPRESSIONS OF EMOTION: STIMULI AND TEST}

The facial expressions of emotion: stimuli and test (FEEST) was used as a standard test of emotion recognition (Young et al., 2002). This is an emotion recognition task, in which subjects need to indicate the emotion that is displayed in a picture of a face, choosing from the six basic emotions (anger, disgust, fear, happiness, sadness, surprise). The task included 60 trials, 10 trials for each of the six basic emotions. Each image was presented during $5 \mathrm{~s}$. 
Table 1 | Characteristics of the pre-symptomatic and symptomatic HD patients and the control group.

\begin{tabular}{|c|c|c|c|}
\hline & $\begin{array}{l}\text { Controls } \\
\text { Mean (SE) }\end{array}$ & $\begin{array}{l}\text { Pre-symptomatic HD } \\
\text { Mean (SE) }\end{array}$ & $\begin{array}{l}\text { Symptomatic HD } \\
\text { Mean (SE) }\end{array}$ \\
\hline Age (years) & $41.0(2.3)$ & $36.2(1.8)$ & $48.8(4.6)$ \\
\hline Gender (F:M) & $13: 9$ & $14: 2$ & $0: 9$ \\
\hline Handedness (R:L) & $21: 1$ & $16: 0$ & 9:0 \\
\hline UHDRS (motor) & - & $1.5(0.3)$ & $31.3(3.0)$ \\
\hline Beck depression inventory & $4.9(1.1)$ & $7.5(1.4)$ & $11.2(0.9)$ \\
\hline Dementia rating scale (total) & $138.0(1.2)$ & $136.3(1.6)$ & $122.6(3.0)$ \\
\hline Rey complex figure (total) & $30.7(0.9)$ & $29.4(1.0)$ & $26.4(1.4)$ \\
\hline Digit symbol - WAIS-III (raw score) & $62.9(4.1)$ & $56.8(3.2)$ & $30.8(3.5)$ \\
\hline Corsi block tapping task (direct) & $48.6(3.9)$ & $46.6(3.6)$ & $31.2(2.8)$ \\
\hline Corsi block tapping task (inverse) & $43.6(5.5)$ & $46.1(3.7)$ & $21.7(4.2)$ \\
\hline Vocabulary - WAIS-III (raw score) & $39.5(2.6)$ & $30.9(3.1)$ & $27.2(4.1)$ \\
\hline Benton visual retention test (total correct) & $6.2(0.4)$ & $5.9(0.4)$ & $3.4(0.6)$ \\
\hline Benton visual retention test (errors) & $5.9(0.7)$ & $6.3(0.8)$ & $12.3(0.9)$ \\
\hline Benton visual form discrimination test (total correct) & $29.7(0.5)$ & $29.8(0.6)$ & $27.3(1.3)$ \\
\hline Benton facial recognition test & $22.6(0.4)$ & $22.6(0.6)$ & $20.6(0.9)$ \\
\hline
\end{tabular}

UHDRS = unified Huntington disease rating scale; WAIS-III= Wechsler adult intelligence scale-third edition.

\section{EMOTION RECOGNITION TASK Material and procedures}

Subjects were tested with an emotion recognition task in which the Ekman faces (Young et al., 2002) were used. Six task conditions were created, one for each of the following emotions: disgust, fear, happiness, sadness, surprise, and anger (see Figure 1C). In each task condition 15 images were shown, of which five target images (displaying the emotion that was tested) and 10 distracter images (displaying other emotions). To control for potential confounds related to the relation between targets and distracters, we used 10 different facial identities during each task condition. Since each task condition contained 15 trials, we had to repeat the facial identity in five trials. We randomly defined whether a target or distracter would be repeated. We also made sure that the 10 distracters included two different faces displaying each of the five distracter emotions. The subject was instructed to indicate for each image whether it displayed the target emotion by pressing one of two buttons on a Cedrus response box. Images were presented during $4 \mathrm{~s}$ and subjects were instructed to respond after the images had disappeared (see Figure 1A). Before each image a fixation cross was presented during $500 \mathrm{~ms}$ to make sure that subjects were always looking at the same point at the beginning of image presentation. Three different task conditions were created in order to study the role of scanning patterns of faces in the emotion recognition deficit found in HD patients.

1. Complete face condition: complete images of faces were shown, displaying one of the six basic emotions. Performance on this task was used to define emotion recognition impairments for each emotion. It was also used to record eye movements (see below).

2. Eyes-only condition: a part of the facial features was masked, in order to define whether this would change recognition of the displayed emotion. In the eyes-only condition, the mouth and nose of each face were masked (for both targets and distracters) and subjects could only use information from the eyes to define the emotion that was displayed (see Figure 1B).

3. Mouth-only condition: this condition was the same as the previous, except that now the eyes region was masked (see Figure 1B).

To further evaluate the scanning pattern to recognize emotions in faces, we recorded eye movements during the first task condition, in which complete faces were used. An iViewX high-speed eye tracker (SMI Neurobehavioral systems) was used. Eye movements were recorded during the $4 \mathrm{~s}$ in which the face stimulus was presented.

\section{Data analyses}

For each task condition, we calculated the number of correctly recognized trials for each emotion separately. To analyze the eye movement data, we selected for each emotion condition those trials in which the target emotion was displayed. Subsequently, the following oculomotor parameters were calculated: number of fixations and fixation duration. To define fixations, we used the BeGaze software, giving priority to saccade detection. Subsequently, three 


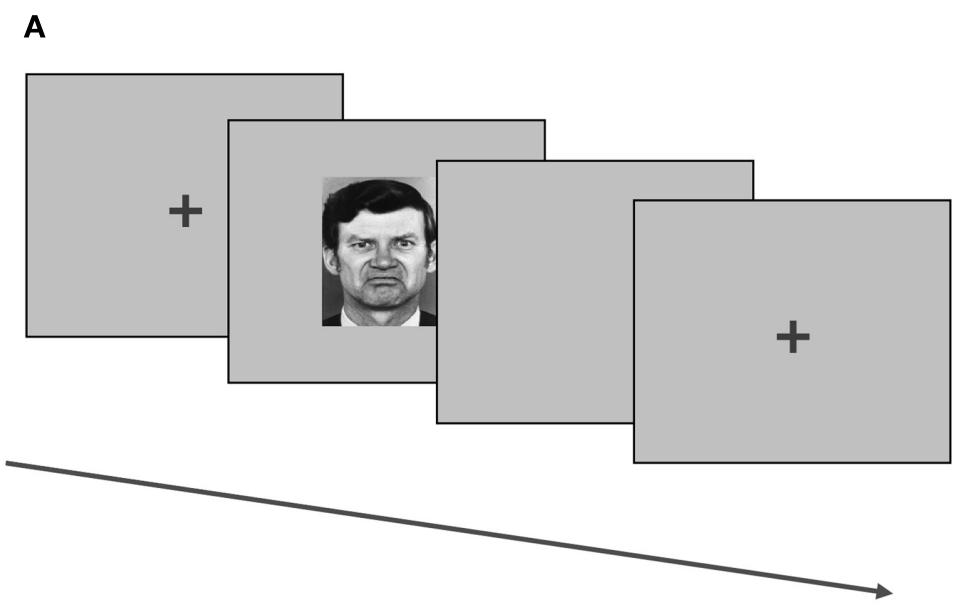

C
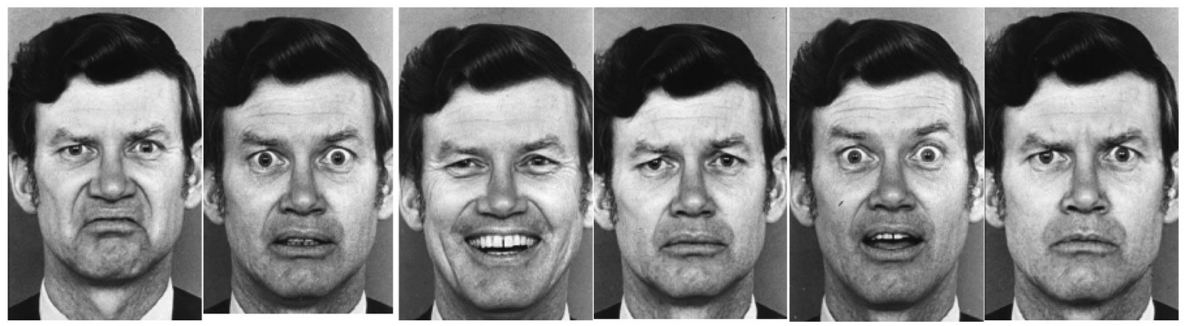

B

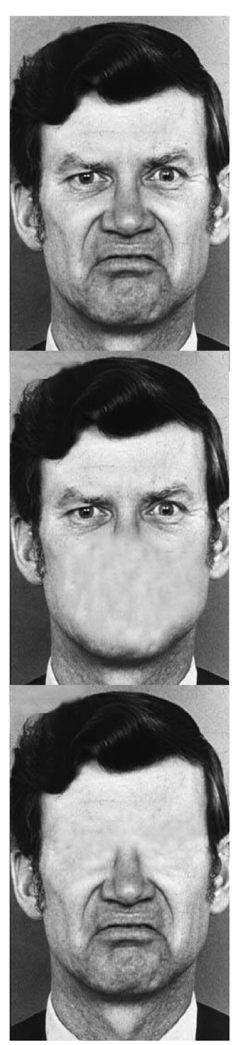

FIGURE 1 | Example of the forced-choice emotion recognition task: (A) task design; (B) example of the three experimental conditions (complete face condition, eyes-only condition, and mouth-only condition); (C) example of the six basic emotions that were used.

regions of interests (ROIs) were used, namely the eyes, the nose, and the mouth. For each of the ROIs we calculated the number of fixations and the total fixation duration.

\section{RESULTS}

\section{BEHAVIORAL RESULTS NEUROPSYCHOLOGICAL TEST BATTERY}

To define whether pre-symptomatic and symptomatic HD patients have cognitive impairments, we compared their performance on a series of neuropsychological tests with healthy control subjects, using separate ANOVAs. We found a significant Group effect for all neuropsychological tasks (all $p$ values $<0.05$ ) that were included, except for the Benton visual form discrimination test $[F(2,44)=2.8]$ and the Benton facial recognition test $[F(2$, $44)=3.1]$. Post hoc analyses indicated that the symptomatic HD patients were impaired on all tasks compared to the control groups $(p<0.05)$, whereas no significant difference was found between the pre-symptomatic HD patients and the control group on any of the tasks.

\section{BEHAVIORAL DATA: FEEST}

Emotion recognition from facial expression was first assessed with the FEEST (Young et al., 2002). Correct responses (see Figure 2) were analyzed using a repeated measures analysis with Emotion (six basic emotions) as within-subject variable and Group (pre-symptomatic and symptomatic HD patients, controls) as between-subjects variable. A significant main effect was found for
Group $[F(2,41)=27.0, p<0.001]$. Post hoc analyses indicated a significant difference between the controls and symptomatic HD patients $(p<0.001)$, but not between the pre-symptomatic HD patients and controls. These findings go against the idea that pre-symptomatic HD patients have a selective impairment for recognition of disgust. Furthermore, an interaction effect was found between Emotion and Group $[F(10,205)=3.5, p<0.001]$. Separate independent-sample $t$-tests including the symptomatic HD patients and controls indicated a significant difference for all emotions (all ps<0.005), except happiness and surprise. These results confirm the findings of Milders et al. (2003), who used the same task.

\section{BEHAVIORAL DATA OF THE TWO-ALTERNATIVE FORCED-CHOICE EMOTION RECOGNITION TASKS}

The number of correctly identified faces (see Figure 3) were analyzed using a repeated measures analyses, including the betweensubject variable Group (pre-symptomatic and symptomatic HD patients, controls) and the within-subject variables Emotion (six basic emotions) and task condition (complete face condition, eyes-only condition, mouth-only condition). A significant main effect for Group was found $[F(2,44)=12.0, p<0.001]$. Post hoc analyses revealed that symptomatic HD patients performed significantly worse than the control group $(p<0.001)$, but not the pre-symptomatic HD gene carriers. An overall interaction effect was found for Emotion $\times$ Group $[F(10,220)=2.1, p<0.05]$. 
Separate ANOVAs were performed for each emotion, indicating that the symptomatic HD patients performed worse than controls

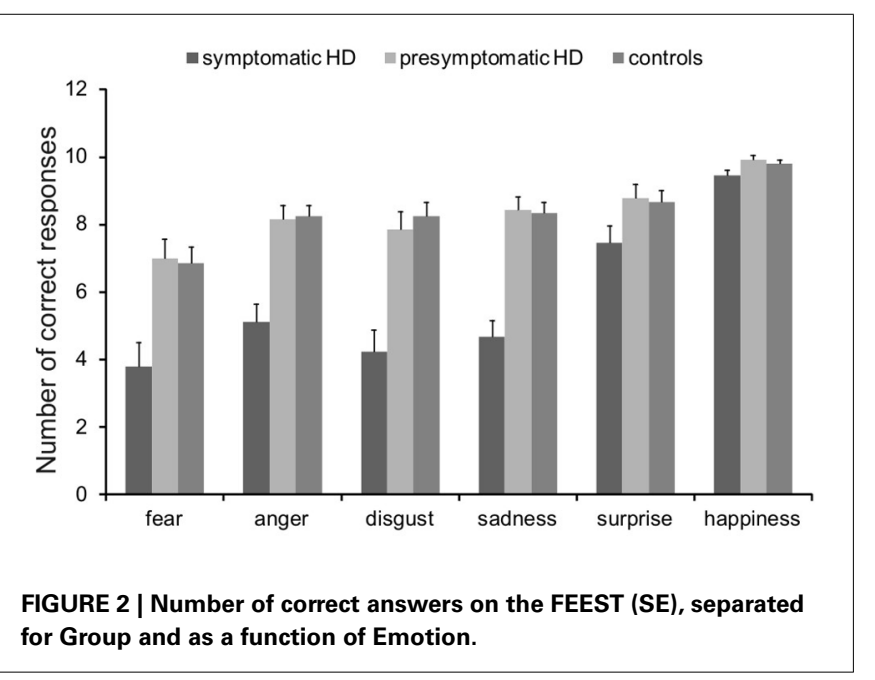

on all emotions. As can be seen in Figure 4 the interaction effect for Emotion $\times$ Group reflects a smaller impairment for the emotion happiness than for the other emotions. No significant interaction for Group $\times$ ROI $\times$ Emotion or for Group $\times$ ROI was found, which means that masking part of the face did not affect the performance of the three groups differently.

\section{EYE MOVEMENTS}

Eye movement data could not be recorded for three presymptomatic HD patients and for two symptomatic HD patients. To define whether pre-symptomatic or symptomatic HD patients have a different way of looking at the eyes, mouth, or nose, we performed a repeated measures analyses, using ROI (eyes, mouth, nose) as within-subject variable and Group (pre-symptomatic, symptomatic HD patients and controls) as between-subject variable. When fixation duration (see Table 2) was used as dependent measure, we found a significant main effect for ROI $[F$ $(2,76)=17.9, p<0.001]$. Contrast analyses indicated a significant difference between the Eyes and Nose region $(p<0.001)$ and between the Eyes and Mouth region $(p<0.001)$, but not
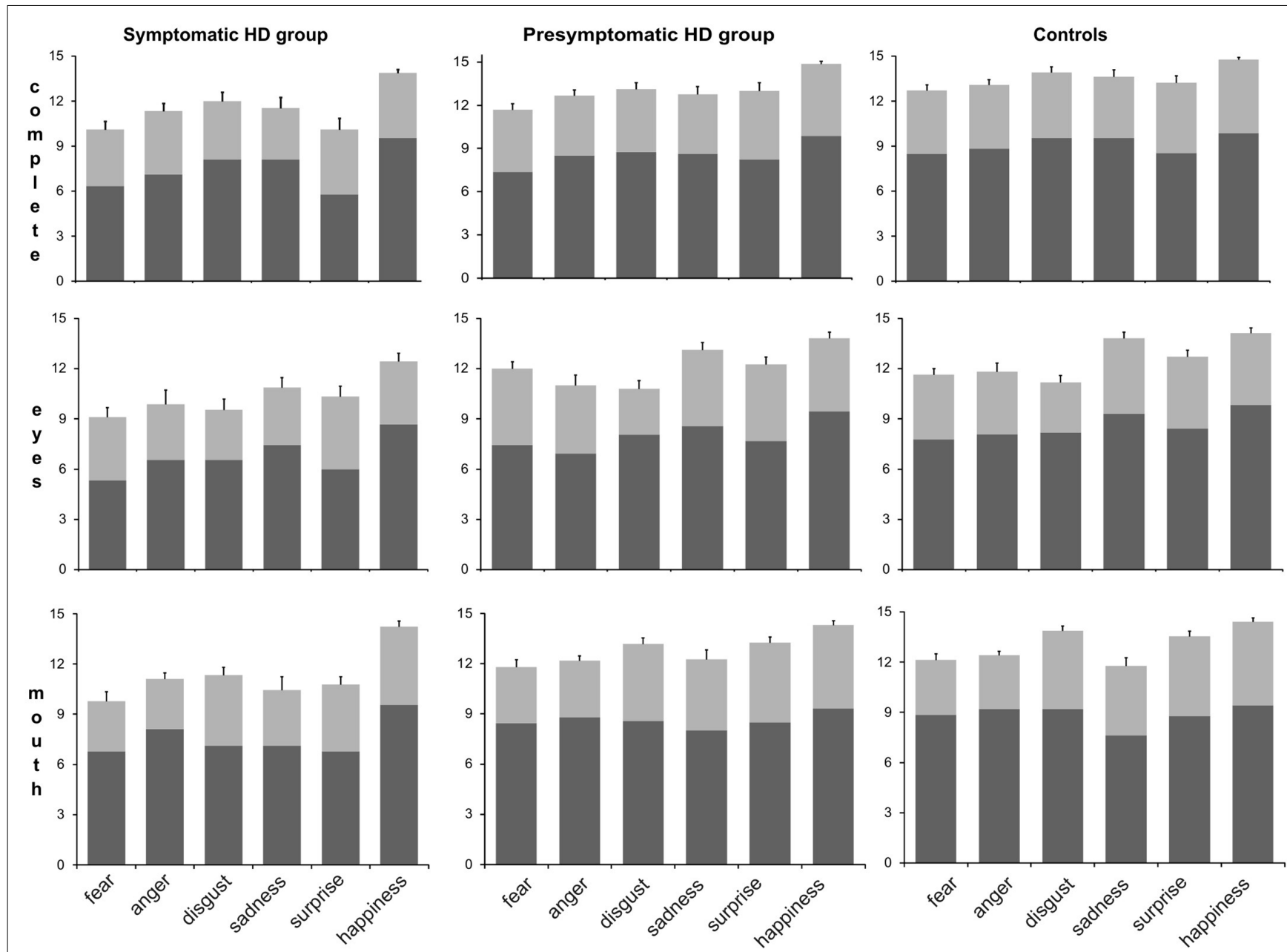

FIGURE 3 | Results of the forced-choice emotion recognition task. Number of correct responses (SE) separated for the three groups, the three tasks conditions, and the six Emotions. Light gray indicates the correctly identified targets and dark gray indicates the correctly identified distracters. 
between the Nose and Mouth region. This means that people look more to the eyes than to the nose and mouth. However, no Group effect was found $[F(2,38)=2.2]$, nor an interaction effect $[F(4,76)=1.3]$. For the number of fixations (see Table 2), a similar pattern was found. A main effect was found for ROI $[F$ $(2,76)=21.7, p<0.001]$. Contrast analyses indicated a significant difference between the eyes and mouth region and the eyes and nose region $(p<0.001)$. No significant difference was found between the nose and mouth region. Importantly, we did not find an interaction effect $[F(4,76)=3.0]$, nor a significant Group effect $[F(2,38)=2.9]$, underlying the previous finding that HD patients and controls make a similar scanning pattern to identify emotions.

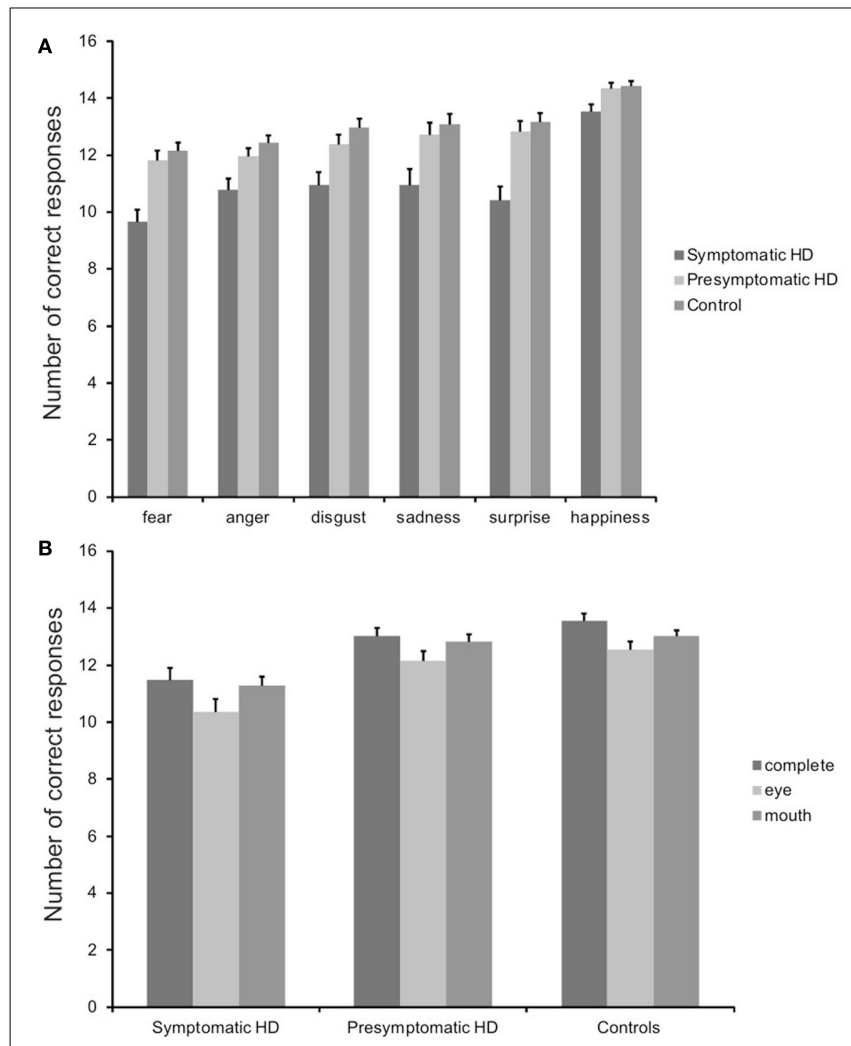

FIGURE 4 | Results of the forced-choice emotion recognition task. (A) Number of correct responses (SE) separated for the three groups and the six emotions; (B) Number of correct responses (SE) separated for the three groups and the three task conditions.

\section{CORRELATION ANALYSES WITH CAG REPEATS}

CAG repeats were correlated with the results of the neuropsychological and emotion recognition tasks, using a two-tailed Pearson correlation. Considering the number of correlations, we used a Bonferroni correction to define statistical significance. We did not find a significant correlation between the CAG repeats on any of the dependent variables for both the pre-symptomatic and symptomatic HD patients, nor when the two groups were taken together.

\section{CORRELATION ANALYSES WITH AGE}

To define the effect of age on emotion recognition performance, we performed a two-tailed correlation analyses between performance on the emotion recognition tasks and age. Considering the number of correlations, we used Bonferroni correction to define statistical significance. No correlation between the different task conditions and age was found for the controls, the pre-symptomatic or symptomatic HD group.

\section{CORRELATION ANALYSES WITH NEUROPSYCHOLOGICAL TESTS}

A two-tailed Pearson correlation was performed between the overall performance on the emotion recognition tests (mean performance on the complete face task condition, eyes-only task condition, and mouth-only task condition) and the neuropsychological tests. A Bonferroni correction was applied. No significant correlation was found for any of the groups on any of the neuropsychological tasks. This suggests that the emotion recognition deficit is not due to an overall cognitive impairment.

\section{DISCUSSION}

The aim of the current study was to investigate the emotion recognition deficit in pre-symptomatic and symptomatic HD patients and to define its underlying mechanism by studying the scanning pattern of faces. Previous studies showed that pre-symptomatic HD patients have a selective deficit in recognition of disgust, whereas recognition of other emotions is unimpaired (Sprengelmeyer et al., 1996; Gray et al., 1997; Hennenlotter et al., 2004). The results of our study could not support this selective deficit. We found that pre-symptomatic HD patients were performing at a normal level on both our two-alternative forced-choice emotion recognition task and the FEEST (Young et al., 2002), which is a test that has often been used to assess emotion recognition in HD. Although several studies have indicated a selective impairment in the recognition of disgust (Sprengelmeyer et al., 1996; Gray et al., 1997; Hennenlotter et al., 2004), this was not supported by all studies (reviewed in Henley et al., 2011). Milders et al. (2003) demonstrated no emotion recognition deficit in pre-symptomatic

Table 2 | Mean fixation duration and number of fixations for the pre-symptomatic and symptomatic HD group and control group separately as a function of ROI.

\begin{tabular}{|c|c|c|c|c|c|c|c|c|c|}
\hline & \multicolumn{3}{|c|}{ Controls } & \multicolumn{3}{|c|}{ Pre-symptomatic HD } & \multicolumn{3}{|c|}{ Symptomatic HD } \\
\hline Fixation duration (ms) & $289(30)$ & $171(19)$ & $145(13)$ & $349(38)$ & $143(25)$ & $127(17)$ & $222(52)$ & $149(34)$ & $133(23)$ \\
\hline
\end{tabular}

SE are mentioned between brackets. 
HD patients, whereas symptomatic HD patients were impaired in recognizing sadness, anger, disgust, and fear. Furthermore, Johnson et al. (2007) found that pre-symptomatic HD patients were significantly impaired on recognition of all negative emotions. Together, these results go against the idea that the basal ganglia, which are affected in pre-symptomatic HD participants, are specifically involved in recognition of disgust.

In contrast, in symptomatic HD patients we found impaired recognition for all emotions when using the two-alternative forced-choice emotion recognition task and on recognition of most emotions when using the FEEST (Young et al., 2002). The results of these tests are in line with previous studies that demonstrated that symptomatic HD patients have an overall emotion recognition impairment affecting most, if not all, emotions (see review Henley et al., 2011). It should be noted that the symptomatic HD patients were also impaired on most of the cognitive tasks that were included in this study. However, since no correlation was found between the performance on the emotion recognition tasks and the cognitive tasks included in the neuropsychological test battery, we can conclude that the emotion recognition deficit is not due to an overall cognitive deficit.

It remains unclear what factors cause the variability in recognition impairment found between the different studies in HD patients. As suggested by Henley et al. (2011), disease variability (e.g., CAG repeat length, disease stage, disease severity) seems to be the most influential factor. In the current study we found that disease stage indeed has a large impact on emotion recognition. That is, whereas pre-symptomatic HD patients were not impaired on any of the emotion recognition tasks, the symptomatic HD patients were impaired on most of the emotion recognition tasks. This indicates that, as the disease progresses, the emotion recognition impairment becomes more pronounced. However, we did not find a correlation between the CAG extension that was defined for each subject and the results of the neuropsychological and emotion recognition tasks. It should be noted, though, that we have included a relatively small group of patients and the range of CAG repeats within these groups is relatively small. Significant correlations with the number of CAG repeats might be found in larger groups of patients with a wider variation in the number of CAG repeats. Another possibility is that the outcome of the various studies on emotion recognition in HD patients is caused by differences in tasks conditions. However, as can be seen in the review of Henley et al. (2011), several studies using the same tasks in different groups of patients have led to different outcomes. In our study we use both a two-alternative forced-choice emotion recognition task and the FEEST, which is a task that has often been used when studying emotion recognition in HD patients. Results of both tasks are largely the same, suggesting that the task that is used does not explain the variability between studies. Finally, we found that age was not associated with performance on the emotion recognition task. Although age can be an indication of the progress of the disease (older people are more likely to be symptomatic than young people), it might also be true that the neuropathology that is found in younger symptomatic HD patients is different from older symptomatic HD patients. To understand which factors define the variability found in emotion recognition impairments in HD patients, a longitudinal study should be performed in which all these factors (number of CAG repeats, disease stage, disease severity, age at onset, task) are well controlled.

To define whether the emotion recognition deficit in symptomatic HD patients is due to impaired visual scanning of faces, we looked at the eye movement patterns during an emotion recognition task. We found that both the pre-symptomatic and symptomatic HD patients spend as much time fixating the eyes, nose, and mouth region as the control subjects do. They also make the same number of fixations to these areas. This indicates that the two patient groups make the same scanning pattern of the different features of a face. These results were confirmed by our second task in which we assessed if performance on the emotion recognition task was altered by showing only part of the facial information. We found that the emotion recognition performance of pre-symptomatic and symptomatic HD patients were similar regardless of whether parts of the faces were deleted or not. These observations suggest that selective scanning of particular face parts does not explain the emotion recognition deficits. These results are in contrast with a recent study in which Blekher et al. (2009) showed that a cognitive impairment in HD patients could partly be explained by a deficit in visual scanning. The fact that we could not replicate this finding using a different cognitive task suggests that the role of oculomotor and visual scanning deficits in cognitive impairments is task dependent. For example, whereas our emotion recognition task requires passive viewing, the digit symbol subscale requires subjects to actively compare digits and symbols and fill in the missing symbols accordingly. The latter task might therefore be more sensitive for the effects of oculomotor deficits. Future studies should be done to further understand the factors that define the role of oculomotor and visual scanning deficits in cognitive impairments found in HD patients. It should be noted that low power might be a concern regarding some trends related to differences in scanning pattern between groups. However this is an unlikely possibility because if there would be a disease related effect, then there should be an order effect ( $\mathrm{HD}<\mathrm{PreHD}<$ Controls). However, this is not what we found $(\mathrm{HD}<$ Controls $<$ PreHD, with all non-significant trends of similar size).

If the emotion recognition deficit that is found in HD patients is not the result of a different scanning pattern of the face, it is more likely the result of higher order processing impairments. These conclusions are in line with recent studies, showing that recognition of disgust is not only impaired in the visual domain, but also in other domains, including auditory perception (Sprengelmeyer et al., 1996; Calder et al., 2010). Furthermore, by studying the effect of contextual information in facial emotion recognition, Aviezer et al. (2009) demonstrated that the HD emotion recognition deficit is not so much due to a low-level perceptual deficit or processing of the general facial configuration, but rather due to impaired explicit recognition of facial expressions. Together, these studies suggest a "cross-modal" emotion recognition deficit (Calder et al., 2010). Apparently, the mechanism underlying the emotion recognition deficit in HD patients is different from the mechanism found in patients with amygdala damage (Adolphs et al., 2005; Kennedy and Adolphs, 2010), who seem to have reduced allocation of attention to the eyes region of the face and therefore have difficulty in recognizing fear. 
Together, the current study reveals two important findings. First, although symptomatic HD patients showed impaired face emotion recognition, we did not find a selective deficit for recognition of disgust in pre-symptomatic HD patients. In fact, presymptomatic HD patients were not impaired on recognition of any of the emotions. Second, we found that both pre-symptomatic and symptomatic HD patients have a normal scanning pattern of the different regions of interest of a face. Thus, the emotion recognition deficit that is found in symptomatic HD patients is not

\section{REFERENCES}

Adolphs, R., Gosselin, F., Buchanan, T. W., Tranel, D., Schyns, P., and Damasio, A. R. (2005). A mechanism for impaired fear recognition after amygdale damage. Nature 433 , 68-72.

Adolphs, R., Tranel, D., Damasio, H., and Damasio, A. (1994). Impaired recognition of emotion in facial expressions following bilateral damage to the human amygdala. Nature 372, 669-672.

Aviezer, H., Bentin, S., Hassin, R. R., Meschino, W. S., Kennedy, J., Grewal, J., Esmail, S., Cohen, S., and Moscovitch, M. (2009). Not on the face alone: perception of contextualized face expressions in Huntington's disease. Brain 132, 1633-1644.

Beck, A. T., Ward, C. H., Mendelson, M., Mock, J., and Erbaugh, J. (1961). An inventory for measuring depression. Arch. Gen. Psychiatry 4, 561-571.

Benton, A. L. (1974). Revised Visual Retention Test, 4th Edn. New York: Psychological Corporation.

Benton, A. L., Hamsher, K., Varney, N. R., and Spreen, O. (1983). Contributions to Neuropsychological Assessment: A Clinical Manual. New York: Oxford University Press.

Berch, D. B., Krikorian, R., and Huha, E. H. (1998). The Corsi block-tapping task: methodological and theoretical considerations. Brain Cogn. 38, 317-338.

Blekher, T., Weaver, M. R., Marshall, J, Hui, S., Gray Jackson, J., Stout, J. C., Beristain, X., Wojcieszek, J., Yee, R. D., and Foroud, T. M. (2009). Visual scanning and cognitive performance in prediagnostic and earlystage Huntington's disease. Mov. Disord. 24, 533-540.

Blekher, T. M., Yee, R. D., Kirkwood, S. C., Hake, A. M., Stout, J. C., Weaver, M. R., and Foroud, T. M. (2004). Oculomotor control in asymptomatic and recently diagnosed individuals with the genetic marker for Huntington's disease. Vision Res. 44, 2729-2736.

Calder, A. J., Keane, J., Manes, F., Antoun, N., and Young, A. W. (2000). Impaired recognition and experience of disgust following brain injury. Nat. Neurosci. 3, 1077-1078.

Calder, A. J., Keane, J., Young, A. W., Lawrence, A. D., Mason, S., and Barker, R. A. (2010). The relation between anger and different forms of disgust: implications for emotion recognition impairments in Huntington's disease. Neuropsychologia 48, 2719-2729.

Cavaco, S., Pinto, C., Gonçalves, A., Gomes, F., Pereira, A., and Malaquias, C. (2008). Auditory verbal learning test: dados normativos dos 21 aos 65 anos (norms for 21 - 65 years old). Psychologica 49, 208-221.

Gómez-Ansón, B., Alegret, M., Muñoz, E., Monté, G. C., Alayrach, E., Sánchez, A., Boada, M., and Tolosa, E. (2009). Prefrontal cortex volume reduction on MRI in preclinical Huntington's disease relates to visuomotor performance and CAG number. Parkinsonism Relat. Disord. 15, 213-219.

Gray, J. M., Young, A. W., Barker, W. A., Curtis, A., and Gibson, D. (1997). Impaired recognition of disgust in Huntington's disease gene carriers. Brain 120, 2029-2038.

Harris, G. J., Codori, A. M., Lewis, R. F., Schmidt, E., Bedi, A., and Brandt, J. (1999). Reduced basal ganglia blood flow and volume in pre-symptomatic, gene-tested persons at-risk for Huntington's disease. Brain 122, 1667-1678.

Henley, S., Novak, M. J. U., Frost, C., King, J., Tabrizi, S. J., and Warren, J. D. (2011). Emotion recognition in Huntington's disease: a systematic review. Neurosci. Biobehav. Rev. 36, 237-253.

Hennenlotter, A., Schoeder, U., Erhard, P., Haslinger, B., Stahl, R., Weindl, A., von Einsiedel, H. G., Lange, K. W., and Ceballos-Baumann, A. O. (2004). Neural correlates associated with impaired disgust processing in pre-symptomatic Huntington's disease. Brain 127, 1446-1453.

Johnson, S. A., Stout, J. C., Solomon, A. C., Langbehn, D. R., Aylward, E. H., Cruce, C. B., Ross, C. A., Nance, M., Kayson, E., Julian-Baros, E., Hayden, M. R., Kieburtz, K., Guttman, M.,

caused by a different scanning pattern of faces. We conclude that there is a general high level emotion recognition deficit that is not explained by impaired scanning pattern of faces in HD.

\section{ACKNOWLEDGMENTS}

This research was supported by a grant from the BIAL Foundation $\left(n^{\circ} 73 / 06\right)$, the Portuguese Foundation for Science and Technology/COMPETE/FEDER (PTDC/PSI-PCO/108208/2008 and PTDC/PSI/67381/2006).

Oakes, D., Shoulson, I., Beglinger, L., Duff, K., Penziner, E., Paulsen, J. S., and the Predict-HD Investigators of the Huntington Study Group (2007). Beyond disgust: impaired recognition of negative emotions prior to diagnosis in Huntington's disease. Brain 130, 1732-1744.

Kennedy, D. P., and Adolphs, R. (2010). Impaired fixation to eyes following amygdala damage arises from abnormal bottom-up attention. Neuropsychologia 48, 3392-3398.

Kessels, R. P. C., Van Zandvoort, M. J. E., Postma, A., Kappelle, L. J., and De Haan, E. H. F. (2000). The Corsi block-tapping task: standardization and normative data. Appl. Neuropsychol. 7, 252-258.

Mattis, S., Jurica, P. J., and Leitten, C. L. (2002). Dementia Rating Scale (DRS2). Lutz, FL: Psychological Assessment Resources.

Milders, M., Crawford, Lamb, A., and Simpson, S. A. (2003). Differential deficits in expression recognition in gene-carriers and patients with Huntington's disease. Neuropsychologia 41, 1484-1492.

Oldfield, R. C. (1971).The assessment and analysis of handedness: the Edinburgh inventory. Neuropsychologia 9, 97-113.

Osterrieth, P. A. (1944). Filetest de copie d'une figure complex: contribution a l' étude de la perception et de la memoire [The test of copying a complex figure: a contribution to the study of perception and memory]. Arch. Psychol. (Geneve) 30, 286-356.

Raven, J. C., Raven, J., and Court, J. H. (1993). Manual for Raven's Progressive Matrices and Vocabulary Scales. Oxford: Oxford Psychologists Press.

Rey, A. (1941). L'examen psychologique dans les cas d'encephalopathie traumatique. Arch. Psychol. 28, 286-340.

Rey, A. (1964). L'examen clinique en psychologie [Clinical Assessment in Psychology]. Paris: Presses Universitaires de France.

Shires, J., Joshi, S., and Basso, M. A. (2010). Shedding new light on the role of the basal gangliasuperior colliculus pathway in eye movements. Curr. Opin. Neurobiol. 20, $6717-725$.

Smith, M. L., Cottrell, G. W., Gosselin, F., and Schyns, P. G. (2005). Transmitting and decoding facial expressions. Psychol. Sci. 16, 3, 184-189.

Sprengelmeyer, R., Schroeder, U., Young, A. W., and Epplen, J. T. (2006). Disgust in pre-clinical Huntington's disease: a longitudinal study. Neuropsychologia 44, 518-533.

Sprengelmeyer, R., Young, A. W., Calder, A. J., Karnat, A., Lange, H., Hömberg, V., Perrett, D. I., and Rowland, D. (1996). Loss of disgust. Perception of faces and emotions in Huntington's disease. Brain 119, 1647-1665.

Thieben, M. J., Duggins, A. J., Good, C. D., Gomes, L., Mahant, N., Richards, F., McCusker, E., and Frackowiak, R. S. J. (2002). The distribution of structural neuropathology in preclinical Huntington's disease. Brain 125, 1815-1828.

Van Asselen, M., Almeida, I., Júlio, F., Januário, C., Bobrowicz Campos, E., Simões, M., Castelo-Branco, M. (in press). Implicit contextual learning in presymptomatic and early stage Huntington's disease patients. J. Int. Neuropsychol. Soc.

Vaz Serra, A., and Pio Abreu, J. L. (1973a). Aferição dos quadros clínicos depressivos. I - Ensaio de aplicação do "Inventário Depressivo de Beck" a uma amostra portuguesa de doentes deprimidos. Coimbra Médica XX, 623-644.

Vaz Serra, A., and Pio Abreu, J. L. (1973b). Aferição dos quadros clínicos depressivos. II - Estudo preliminar de novos agrupamentos sintomatológicos para complemento do "Inventário Depressivo de Beck." Coimbra Médica XX, 713-736.

Wechsler, D. (1997). WAIS-III: Administration and Scoring Manual. San Antonio: The Psychological Corporation.

Wechsler, D. (2008). WAIS-III: Escala de Inteligência de Wechlser para Adultos -3. a edição: Manual [Wechsler Adult Intelligence Scale (WAIS-III)]. Lisboa: Cegoc. 
Young, A. W., Perret, D. I., Calder, A., Sprengelmeyer, R., and Ekman, P. (2002). Facial Expressions of Emotion: Stimuli and Test. San Antonio, TX: Harcourt Assessment.

Conflict of Interest Statement: The authors declare that the research was conducted in the absence of any commercial or financial relationships that could be construed as a potential conflict of interest.

Received: 14 June 2011; accepted: 27 January 2012; published online: 16 February 2012.

Citation: van Asselen $M$, Júlio $F$ Januário C, Campos EB, Almeida I,
Cavaco S and Castelo-Branco M (2012) Scanning patterns of faces do not explain impaired emotion recognition in Huntington disease: evidence for a high level mechanism. Front. Psychology 3:31. doi: 10.3389/fpsyg.2012. 00031

This article was submitted to Frontiers in Emotion Science, a specialty of Frontiers in Psychology.
Copyright (C) 2012 van Asselen, Júlio, Januário, Campos, Almeida, Cavaco and Castelo-Branco. This is an openaccess article distributed under the terms of the Creative Commons Attribution Non Commercial License, which permits non-commercial use, distribution, and reproduction in other forums, provided the original authors and source are credited. 